\title{
Saúde auditiva e qualidade de vida em trabalhadores expostos a agrotóxicos
}

\author{
Hearing care and quality of life \\ among workers exposed to pesticides
}

Tereza Raquel Ribeiro de Sena ${ }^{1}$

Marlizete Maldonado Vargas ${ }^{2}$

Cristiane Costa da Cunha Oliveira ${ }^{3}$

${ }^{1}$ Centro de Ciências Biológicas e da Saúde, Universidade Federal de Sergipe. Av. Mal Rondon, Rosa Elze. 49100-000 São Cristóvão SE. tr@trsena.com.br ${ }^{2}$ Mestrado em Saúde Ambiente, Curso de Psicologia, Universidade Tiradentes.

${ }^{3}$ Mestrado em Saúde e Ambiente, Universidade Tiradentes.

\begin{abstract}
The objective of this study was to determine the relationship between exposure to pesticides and the occurrence of hearing loss among rural workers of Povoado Colonia Treze, Lagarto, State of Sergipe, Brazil. A total of 351 adult workers aged 18 to 59 years were selected, with current or past work activity in rural areas, with or without use of pesticides. The hearing evaluation included a form to record air-borne tonal thresholds using normality criteria recommended by Merluzzi 1979. The instrument used for assessing the quality of life was the Brazilian version of Short Form 36 (SF-36). Statistical analysis was performed (chi-square $c^{2}$, nonparametric Kruskal-Wallis test, Cramer's V and Cohen's d) with a significance level of $95 \%(p<0.05)$. The results related the use of pesticide with the degree of toxicity in the presence of hearing loss and indices of quality of life $(p<0.001)$. This study proved that agricultural workers, users of pesticides showed the worst levels of quality of life when compared with those who have not handled them. The use of pesticides and their toxicity class interfered in a most striking way in the classification of hearing loss presented in this group and further studies are recommended to assess other impacts on this population.
\end{abstract}

Key words Pesticides, Occupational health, $R u$ ral population, Quality of life
Resumo O objetivo deste estudo foi determinar a relação entre a exposição ao agrotóxico e a ocorrência de perda auditiva nos trabalhadores rurais do Povoado Colônia Treze, Lagarto (SE). Foram selecionados 351 trabalhadores adultos na faixa etária de 18 a 59 anos, com atividade laboral atual ou pregressa na área rural, com ou sem uso de agrotóxico. A avaliação audiológica foi composta de ficha para registro dos limiares tonais aéreos pelo critério de normalidade preconizado por Merluzzi 1979. O instrumento usado para a avaliação da qualidade de vida foi a versão brasileira do Short Form 36 (SF-36). Foi realizada análise estatística (testes qui-quadrado $\chi^{2}$, não paramétrico de Kruskal-Wallis, $V$ de Cramer ed de Cohen) com nível de significância de 95\% $(p<0,05)$. Os resultados relacionaram o uso de agrotóxico com o grau de toxicidade, com a presença de perda auditiva e com os indices de qualidade de vida ( $p$ $<0,001)$. Este estudo pode comprovar que os agricultores, usuários de agrotóxicos apresentaram piores níveis de qualidade de vida quando comparados com aqueles que não os utilizaram. O uso de agrotóxico e sua classe toxicológica interferiram de maneira mais impactante na classificação de perda auditiva apresentada nos mesmos e novos estudos são recomendados para avaliar outros impactos nesta população.

Palavras-chave Praguicidas, Saúde do trabalhador, População rural, Qualidade de vida 


\section{Introdução}

A população mundial sofre um crescimento acelerado e utiliza estratégias para suprir o aumento do consumo de alimentos com a necessidade de grandes propriedades, maquinários e emprego de produtos químicos como agrotóxicos e fertilizantes que podem repercutir em agravos à saúde e ao meio ${ }^{1}$. Neste contexto, os agrotóxicos são considerados importantes fatores de risco tanto à saúde dos trabalhadores quanto ao ambiente.

As repercussões à saúde dos trabalhadores rurais e à comunidade circunvizinha exposta aos agrotóxicos representam um problema de saúde pública alvo de ações governamentais, no intuito de atuar de forma integrada na promoção da saúde, na prevenção dos agravos e na participação e controle social ${ }^{2}$. Os efeitos colaterais ocasionados pelo consumismo desenfreado da sociedade e as novas tecnologias utilizadas comprometem a saúde do planeta e a relação harmoniosa do homem com o meio, o que implicará numa qualidade de vida limitada, pois a exemplo dos alimentos mais resistentes pelo uso de agrotóxicos, esta nocividade não é totalmente conhecida ${ }^{3}$.

Os efeitos deletérios à saúde humana são descritos por alterações nos sistemas nervoso, cardiovascular, respiratório, na pele, nos olhos, além de alterações hematológicas e reações alérgicas aos agrotóxicos ${ }^{4,5}$. Além disso, a sintomatologia apresentada nos casos de intoxicação por agrotóxicos pode ser confundida com outros agravos, pois os trabalhadores não associam sintomas como astenia, náuseas, vômitos, cefaleia, dificuldade respiratória e dores abdominais à exposição a este agente de risco ${ }^{6,7}$.

Entretanto, a magnitude das intoxicações por agrotóxicos não é totalmente conhecida e o controle da morbi-mortalidade decorrente desta exposição apresenta-se dificultado pelos dados discrepantes entre as notificações do sistema nacional de agravos de notificação (Sinan) e do sistema nacional de informações tóxico-farmacológicas (Sinitox) que alimentam a base de dados da Anvisa $^{6,8}$. Da mesma forma que as misturas de produtos diferentes e o uso de venenos contrabandeados dificultam a análise dos efeitos reais à saúde e ao ambiente ${ }^{6}$.

Os agrotóxicos são utilizados em larga escala no Brasil e mais intensamente no setor rural, a agricultura ${ }^{4}$. De acordo com a Agência Nacional de Vigilância Sanitária (Anvisa), órgão de normatização, controle, regulação e fiscalização da vigilância sanitária brasileira, os agrotóxicos são classificados quanto à toxicidade em: classe I (ex- tremamente tóxico), classe II (altamente tóxicos), classe III (moderadamente tóxicos) e classe IV (pouco tóxicos). Porém esta classificação se relaciona mais diretamente à intoxicação aguda e não indica os riscos de doenças de evolução prolongada, de acometimento crônico, como, por exemplo, câncer, neuropatias, hepatopatias, problemas respiratórios crônicos e outros.

O uso de agrotóxicos pode ter repercussões importantes na qualidade de vida daqueles que os manipulam. O conceito abrangente de qualidade de vida preconizado pela Organização Mundial de Saúde incorpora de uma forma complexa, a saúde física, o estado psicológico, o nível de dependência, as relações sociais, as crenças pessoais e o relacionamento com características que se destacam no ambiente?.

Além das implicações sobre a saúde geral e a qualidade de vida dos agricultores expostos a agrotóxicos, alguns produtos químicos presentes no processo produtivo podem ser nocivos à audição. Assim, a perda auditiva pode representar um sinal precoce de intoxicação ao agrotóxico ${ }^{10-}$ ${ }^{12}$. No tocante à perda auditiva ocasionada por ototóxicos, esta se apresenta, em geral, como perda do tipo sensório-neural bilateral simétrica, de caráter irreversível seletiva em altas frequências $(3 \mathrm{~K} \mathrm{a} 6 \mathrm{KHz})$ de configuração em entalhe por lesão principalmente em células ciliadas da cóclea ${ }^{13}$.

O Estado de Sergipe quanto ao ramo de atividade, apresenta um grande número de trabalhadores no setor agrícola, com importante relevância no perfil socioeconômico estadual. O segundo maior grupo econômico agropecuário do Estado é a agricultura, com aproximadamente 34.468 lavouras temporárias ${ }^{14,15}$. Dentre as atividades agrícolas se destacam o cultivo de laranja, cana-de-açúcar, coco e fumo para exportação, desta forma, há um grande número de trabalhadores neste ramo de atividade ${ }^{16}$. O trabalho rural é significativamente mais perigoso que outras atividades e estima-se que muitos agricultores sofram sérios problemas de saúde ${ }^{17}$.

Nesse contexto, a relevância do presente estudo epidemiológico de base populacional se encontra no fato de que a investigação do papel dos agrotóxicos como agente de risco ocupacional esteja relacionado com os danos à saúde auditiva e à qualidade de vida dos trabalhadores rurais. Desta forma, sendo considerado um problema de saúde coletiva com impactos sobre a sociedade e o ambiente. Esta pesquisa teve como objetivo determinar a relação entre a exposição ao agrotóxico e a ocorrência de perda auditiva nos trabalhadores rurais do Povoado Colônia 
Treze, Lagarto (SE) e verificar o impacto do uso de agrotóxicos sobre sua qualidade de vida. Como objetivos específicos estabeleceram-se verificar a prevalência do uso de agrotóxico pelos trabalhadores rurais pesquisados e o grau de toxicidade dos mesmos pelo critério de classificação toxicológica preconizado pela Anvisa; avaliação da ocorrência de perda auditiva nos trabalhadores expostos e o nível de qualidade de vida dos sujeitos, bem como, determinar possíveis associações entre qualidade de vida e exposição ao agrotóxico.

\section{Métodos}

\section{Delineamento do estudo}

Esta pesquisa é um estudo de caráter observacional transversal e foi realizada entre os meses de novembro de 2010 e abril 2011.

\section{Local do estudo}

Este estudo foi realizado no Povoado Colônia Treze, localizado no município de Lagarto, porção sul do Estado de Sergipe, a 78 km de distância da capital deste, Aracaju, região nordeste do Brasil.

\section{População do estudo}

O Povoado Colônia Treze possui 4.936 habitantes e 1.353 domicílios, caracterizando-se como o maior povoado sergipano em número de habitantes do Estado e possui atividade predominantemente rural no cultivo de fumo e de cítricos entre outras culturas ${ }^{18}$.

\section{Seleção da amostra}

O cálculo amostral tomou como parâmetro a distribuição populacional por faixa etária de acordo com o censo agropecuário do Instituto Brasileiro de Geografia e Estatística (IBGE) ${ }^{14}$. Foi realizado plano amostral por conglomerado para cálculos do tamanho da amostra e foi utilizada a fórmula de Barbetta com proporções adequadas ao percentual por faixas etárias totalizando 352. Neste estudo foram incluídos 351 trabalhadores adultos na faixa etária de 18 a 59 anos, de ambos os gêneros, com atividade laboral atual ou pregressa associada ao setor agrícola, na área rural, com ou sem uso de agrotóxico. Todos os sujeitos que se dispuseram a participar da pes- quisa e possuíam vínculo de trabalho formal, informal e/ou atuavam na agricultura familiar rural no Povoado Colônia Treze foram incluídos. Os trabalhadores que não contemplaram estas características e um trabalhador de 19 anos que havia sofrido acidente automobilístico, apresentando saúde geral muito debilitada, comparada com os demais, foram excluídos.

\section{Aspectos éticos}

O projeto atendeu os aspectos éticos de pesquisa com seres humanos tendo sido submetido e aprovado pelo Comitê de Ética em Pesquisa da Universidade Tiradentes (Unit), não havendo conflito de interesses. Todos os sujeitos que concordaram em participar do estudo assinaram o termo de consentimento livre e esclarecido previamente lido e explicado.

\section{Procedimentos}

Inicialmente, foi realizada uma capacitação prévia com uso de recursos visuais, aos agentes comunitários de saúde da Prefeitura Municipal de Lagarto que realizavam a cobertura de saúde da família no Povoado, cujo conteúdo versava sobre riscos à saúde do trabalhador rural, saúde auditiva, qualidade de vida, apresentação do projeto de pesquisa e cronograma.

Posteriormente, a equipe de pesquisadores também inserida no projeto de extensão Quali 13 - Projeto de qualidade de vida e saúde nos trabalhadores rurais da Colônia Treze, participou em conjunto da coleta de dados.

A avaliação audiológica foi precedida da mensuração do nível de pressão sonora existente na sala para confirmação das intensidades do local a fim de certificar que este se apresentava em consonância com a norma ISO 8.253-1. Foi utilizado medidor de nível sonoro DEC 490, IEC tipo II, na faixa 30 a $130 \mathrm{~dB}$, com circuito de ponderação "A", tempo de integração slow, resolução de $0,1 \mathrm{~dB}$, com protetor de vento. Posteriormente, foi preenchida a ficha de caracterização do sujeito da pesquisa com o roteiro de anamnese audiológica ocupacional, perguntas sobre idade e sexo do sujeito; o tempo e a forma de trabalho rural, se possuía vínculo de trabalho formal, na agricultura familiar ou outro; qual o tipo de cultura plantada; se fazia uso de agrotóxicos e quais os utilizados; se utilizava ou não equipamentos de proteção individual (EPI); se havia a exposição a outros agentes risco ocupacional e registrava-se o resultado da meatoscopia. O examinador fez 
uso de luvas descartáveis durante este procedimento como também utilizou espéculos individuais higienizados com água corrente, sabão e álcool a 70\%. A audiometria tonal liminar pesquisou a via aérea de ambas as orelhas nas frequências de $1 \mathrm{~K}, 2 \mathrm{~K}, 3 \mathrm{~K}, 4 \mathrm{~K}, 6 \mathrm{~K}$ e $8 \mathrm{~K} \mathrm{~Hz}$ e o audiômetro de 1 canal Amplivox A260 com fones TDH-39, se encontrava calibrado com certificado no prazo de validade em conformidade com a legislação vigente.

Todos os exames audiológicos foram catalogados e classificados como normo-ouvintes Grau 0 segundo critério de Merluzzi et al. ${ }^{19}$; hipoacúsicos com exposição a agrotóxico (perdas auditiva seletiva em frequências altas) - Grau 1; hipoacúsicos com exposição a agrotóxico e com exposição a outro agente de risco auditivo (perda auditiva acompanhada de outro agente de risco) - Grau 2; e hipoacúsicos sem exposição a agrotóxico (perdas auditivas sem exposição a agrotóxico) - Grau 3.

$\mathrm{Na}$ avaliação da qualidade de vida, utilizou-se ficha da versão brasileira do questionário de qualidade de vida Short Form 36 (SF-36). O SF-36 foi composto de 11 questões e 36 itens que contemplaram 8 domínios: capacidade funcional (dez itens), aspectos físicos (quatro itens), dor (dois itens), estado geral de saúde (cinco itens), vitalidade (quatro itens), aspectos sociais (dois itens), aspectos emocionais (três itens), saúde mental (cinco itens) e uma questão sobre a percepção atual da saúde. A aplicação do questionário SF36 ocorreu no mesmo consultório da avaliação audiológica ou em outra sala, se realizada por mais de um examinador. Cada sujeito pesquisado teve seus pontos por questão somados e após a aplicação da fórmula para cálculo de cada domínio recebeu um escore por domínio que variou de zero, pior resultado, a 100, melhor resultado ${ }^{20}$.

\section{Análise dos Dados}

Os dados referentes aos indivíduos pesquisados foram analisados quanto à exposição a agrotóxico, quanto à classe toxicológica dos agrotóxicos, quanto à prevalência e ao grau de perda auditiva e quanto aos índices de qualidade de vida de acordo com os domínios do SF-36. Foi calculada a prevalência de perda auditiva na população estudada e realizada a análise da variável dependente uso de agrotóxico de acordo com gênero, tipo de perda auditiva e grau de toxicidade do agrotóxico.

Foi aplicado o teste estatístico $\chi^{2}$ (qui-quadrado) para verificação de existência de possíveis diferenças entre os grupos pesquisados, expostos e não expostos, quanto à presença e tipo de perda auditiva de acordo com o grau de toxicidade dos agrotóxicos utilizados pelos trabalhadores (variáveis categóricas). O nível de significância adotado foi de 95\% $(p<0,05)$. Para a análise dos parâmetros do questionário SF-36 foi utilizado o teste não paramétrico de KruskalWallis. Para o cálculo do tamanho do efeito, ou seja, da magnitude compreendida como relação da força de associação, calculado exclusivamente para as variáveis que denotaram significância estatística $(p<0,001)$, foram utilizados o teste $\mathrm{V}$ de Cramer para variáveis categóricas com mais de duas variáveis e o teste d de Cohen para variáveis contínuas em escala ordinal ou intervalar.

\section{Resultados e discussão}

A distribuição do plano amostral por sexo indicou que 51,9\% (182) eram homens e 48,1\% (169) mulheres $^{21}$. A média de idade dos participantes foi de 33 anos ( $D P=12,045)$ e a média do tempo de atividade laboral foi de 16 anos. A agricultura familiar se apresentou em maior número $(\mathrm{n}=$ 274) que as relações de trabalho formais $(n=77)$.

Do total de trabalhadores rurais avaliados $67,0 \%(\mathrm{n}=235)$ faziam uso de agrotóxico e destes $71,9 \%$ não utilizavam proteção individual (n =169). Dentre as culturas, o cultivo de fumo e outras culturas representaram $71,5 \%(n=251)$ e apenas $28,5 \%(\mathrm{n}=100)$ não plantavam fumo. Estes percentuais comparam as características locais do município de Lagarto como maior produtor da cultura de fumo de Sergipe $(78,1 \%)^{22,23}$.

Apenas um sujeito não usuário de agrotóxico usava proteção individual tais como máscara, camisa de mangas compridas, calça e chapéu com aba, por medida de segurança no período em que o vizinho aplicava o agrotóxico.

Quanto à classe toxicológica dos agrotóxicos utilizados, a maior distribuição, exceto aqueles sem classificação, corresponde a 161 sujeitos que desconheciam a classe ou não estavam expostos, seguida da classe toxicológica IV (pouco tóxicos), com 89 usuários (25,4\%), e da classe I (extremamente tóxicos), 64 usuários $(18,2 \%)$.

A prevalência de alteração meatoscópica foi de $15,7 \%$ nos pesquisados, sendo maior que o estabelecido para as alterações na orelha externa em adultos, a saber, de $2 \%$ a $6 \%$ da população ${ }^{24}$. Em relação à exposição a outro agente de risco auditivo, 77,5\% não possuía histórico de exposição a ruído. Os normo-acúsicos representaram 
a maioria dos pesquisados, perfazendo um total de $63,8 \%$ e os classificados como hipoacúsicos com exposição a agrotóxico, 23,4\%. Os demais ficaram distribuídos em hipoacúsicos sem exposição a agrotóxico $(6,6 \%)$ e com hipoacusia com exposição a agrotóxico somado a outro agente de risco $(6,3 \%)$, conforme Tabela 1.

Um estudo de Manjabosco et al. ${ }^{7}$ numa amostra de 42 sujeitos verificaram a prevalência de transtornos auditivos em $60 \%$ de agricultores examinados, porém todos apresentavam histórico de exposição a mais de um tipo de ruído; entretanto, não foi observada nenhuma relação direta com o uso de agrotóxicos visto que nem todos tiveram contato direto com este agente de risco.

A maioria dos indivíduos que utilizavam agrotóxicos da classe toxicológica IV (pouco tóxico) eram mulheres $(55,6 \%)$ sendo a diferença significativa quando comparada com os homens $(22,8 \% ; p<0,001)$. O uso de agrotóxico da classe toxicológica I (extremamente tóxico) foi mais comum em homens $(56,7 \%)$ comparado com o uso por mulheres $(27,0 \%)$ como apresentado na Tabela 2. Este resultado pode ser deduzido, pois a população de agricultores do sexo masculino é geralmente provedora da família e tem se exposto a agrotóxicos perigosos à saúde. Este resultado está em consonância com os estudos de Damalas e Eleftherohorinos ${ }^{25}$, Silva et al. ${ }^{26}$ e Soares et al. ${ }^{27}$ nos quais ficou evidente que os homens usavam mais agrotóxicos que as mulheres sendo que a mistura de diversos tipos de agrotóxicos poderia potencializar os prejuízos à saúde. Salienta-se que este processo é uma rotina, se mostrando uma situação muito presente na realidade do trabalho agrícola por causa do uso simultâneo de várias substâncias.

A maioria dos indivíduos (97,6\%) com exposição a agrotóxico apresentou classificação audiométrica de grau I (hipoacusia com exposição a agrotóxico). Quanto à classe toxicológica, os que utilizavam agrotóxico de maior toxicida- de, classe I (66,2\%), a mais comum para a maioria dos agricultores que foram classificados como grau I, ou seja, hipoacúsicos com exposição a agrotóxico $(p<0,001)$, como disposto na Tabela 3. A exemplo dos achados de Teixeira et al. ${ }^{17}$, que observaram em 57,14\% de 98 trabalhadores expostos a pesticidas, no controle de endemias vetoriais em Pernambuco, sinais de perdas auditivas nas altas frequências. Porém, destaca-se que a força de associação entre a classificação audiométrica e o uso de agrotóxico (V de Cramer 0,489), o tipo de classe toxicológica (V de Cramer 0,307$)$ foi maior que os demais aspectos pesquisados que apresentaram significância estatística (Tabela 4).

Em relação ao tempo de atividade laborativa no trabalho rural entre os que usavam e não usa-

Tabela 2. Distribuição da classe toxicológica dos agrotóxicos por gênero dos agricultores usuários, Colônia Treze, SE, 2010-11.

\begin{tabular}{cccc}
\hline Classe toxicológica & \multicolumn{3}{c}{ Gênero dos pesquisados } \\
\cline { 2 - 4 } dos agrotóxicos & Masculino & Feminino & Total \\
\hline Classe IV & & & \\
$\mathrm{n}$ & 29 & 35 & 64 \\
$\%$ & 22,8 & 55,6 & 33,78 \\
Classe III & & & \\
$\mathrm{n}$ & 12 & 6 & 18 \\
$\%$ & 9,4 & 9,5 & 9,5 \\
Classe II & & & \\
$\mathrm{n}$ & 14 & 5 & 19 \\
$\%$ & 11,0 & 7,9 & 10,0 \\
Classe I & & & \\
$\mathrm{n}$ & 72 & 17 & 89 \\
$\%$ & 56,7 & 27,0 & 46,8 \\
Total & & & \\
$\mathrm{n}$ & 127 & 63 & 190 \\
$\%$ & 100 & 100 & 100 \\
\hline
\end{tabular}

$\mathrm{X}^{2}=21,721(\mathrm{p}<0,001)$

Tabela 1. Distribuição dos agricultores de acordo com classificação audiométrica, Colônia Treze, SE, 2010-11.

\begin{tabular}{lcrr}
\hline \multicolumn{1}{c}{ Classificação audiométrica } & Grau & n & \% \\
\hline & 0 & 224 & 63,8 \\
Normoacusia & 1 & 82 & 23,4 \\
Hipoacusia com exposição a agrotóxico & 2 & 22 & 6,3 \\
Hipoacusia com exposição a agrotóxico e outro agente de risco à audição & 3 & 23 & 6,6 \\
Hipoacusia sem exposição a agrotóxico & Total & 351 & 100,0 \\
& &
\end{tabular}


Tabela 3. Distribuição toxicológica dos agrotóxicos de acordo com a avaliação audiométrica dos agricultores. Colônia Treze, SE, 2010-11.

\begin{tabular}{lrrrr}
\hline \multicolumn{1}{c}{ Classe toxicológica dos agrotóxicos } & Grau $\mathbf{0}$ & Grau 1 & Grau 2 & Total \\
\hline Classe IV & & & & \\
n & 47 & 14 & 3 & 64 \\
$\%$ & 46,1 & 20,6 & 11,1 & 33,7 \\
Classe III & & & & \\
n & 10 & 3 & 5 & 18 \\
$\%$ & 9,8 & 4,4 & 27,8 & 9,5 \\
Classe II & & & & \\
n & 12 & 6 & 1 & 19 \\
$\%$ & 11,7 & 8,8 & 5,6 & 10,0 \\
Classe I & & & & \\
n & 33 & 45 & 11 & 89 \\
$\%$ & 32,4 & 66,2 & 55,6 & 46,8 \\
Total & & & & \\
n & 102 & 68 & 20 & 190 \\
$\%$ & 100,0 & 100,0 & 100,0 & 100,0 \\
\hline
\end{tabular}

$\mathrm{X}^{2}=30,662 ;(\mathrm{p}<0,001)$

Tabela 4. Força de relação entre classificação audiométrica e as variáveis com significância estatística. Colônia Treze, SE, 2010-11.

\begin{tabular}{lcr}
\hline \multicolumn{1}{c}{$\begin{array}{c}\text { Classificação } \\
\text { audiométrica versus }\end{array}$} & \multicolumn{2}{c}{ Testes estatísticos } \\
\cline { 2 - 3 } & $\begin{array}{r}\text { V de } \\
\text { Cramer }\end{array}$ & $\begin{array}{r}\mathbf{d ~ d e} \\
\text { Cohen }\end{array}$ \\
\hline Gênero & 0,284 & \\
Uso de agrotóxico & 0,489 & \\
Classe toxicológica & 0,307 & \\
Meatoscopia & 0,246 & \\
Tipo de cultura & 0,273 & \\
Idade & & 0,207 \\
Tempo de trabalho & & 0,212 \\
Capacidade funcional & & 0,055 \\
Aspectos físicos & & 0,090 \\
Dor & & 0,096 \\
Vitalidade & & 0,139 \\
EGS & & 0,180 \\
\hline
\end{tabular}

vam agrotóxicos houve diferença significativa nessa relação $(p<0,001)$, ou seja, indivíduos com mais tempo de trabalho rural (20 anos) usavam agrotóxicos com mais frequência quando comparados aos com menor tempo de trabalho (10 anos), o que pode indicar uma naturalização do uso de agrotóxicos percebidos a partir de duas concepções antagônicas, ou seja, como remédio e veneno, na medida em que, sendo percebido como "remédio", o agrotóxico era usado para garantir a produtividade das lavouras mesmo com a possibilidade de provocar efeitos adversos à saúde ${ }^{21}$.

Na definição de papéis impostos ao trabalhador rural no âmbito familiar, este necessita de constante força física para o trabalho, o adoecimento pode sobrecarregar outros membros e comprometer a subsistência da família ${ }^{28}$, desta forma, ao analisar os aspectos da qualidade de vida do grupo populacional estudado, foram evidenciados (Tabela 5) escores nos domínios dor e vitalidade em trabalhadores expostos (84 e 75) menores do que naqueles que não foram expostos a agrotóxicos (100 e 90). No tocante à interferência nos aspectos sociais, os agricultores usuários de agrotóxicos $(\mathrm{x}=75)$ apresentaram maior tendência a ter dificuldades nas relações sociais do que aqueles que não usavam agrotóxicos ( $\mathrm{n}=$ $100, p=0,019)$. Nesse contexto, o trabalho na agricultura estaria vinculado às relações sociais que estruturam a família, envolvem valores, diferenciações de papéis e hierarquias que podem se mostrar conflituosas quando comparadas entre os indivíduos que fazem e os que não fazem uso de agrotóxicos pelos danos oriundos da exposição a este agente de risco. Estes achados guardam relação quando associados à utilização de agrotóxico com o escore total de qualidade de vida do SF-36. Desta forma, os indivíduos que foram expostos a agrotóxicos apresentaram escores de qualidade de vida significativamente inferiores em todos os domínios, a exceção da capacidade funcional, quando comparados a indivíduos não expostos $(p=0,024)$. Ou seja, os agricultores que se 
Tabela 5. Distribuição dos agricultores usuários e não usuários de agrotóxicos do Povoado Colônia Treze, SE, 2010-11 de acordo com a idade, tempo de trabalho rural e domínios do SF-36.

\begin{tabular}{lcccccc}
\hline & Uso de agrotóxico & $\mathbf{n}$ & Média & $\mathbf{U}$ & p-valor & Medianas X \\
\hline Idade do agricultor & Não & 116 & 133,51 & $8.701,00$ & $<0,001$ & 25,00 \\
\multirow{3}{*}{ Tempo de trabalho rural } & Sim & 235 & 196,97 & & & 35,00 \\
\multirow{3}{*}{ Aspectos físicos } & Não & 116 & 125,08 & $7.723,50$ & $<0,001$ & 10,00 \\
& Sim & 235 & 201,13 & & & 20,00 \\
Limitação por aspectos emocionais & Não & 116 & 197,09 & $11.183,50$ & 0,003 & 100,00 \\
& Sim & 235 & 165,59 & & & 90,00 \\
Aspectos sociais & Não & 116 & 198,84 & $10.980,50$ & 0,001 & 100,00 \\
& Sim & 235 & 164,73 & & & 66,00 \\
Dor & Não & 116 & 193,38 & $11.613,50$ & 0,019 & 100,00 \\
\multirow{2}{*}{ Vitalidade } & Sim & 235 & 167,42 & & & 75,00 \\
\multirow{2}{*}{ Saúde mental } & Não & 116 & 202,69 & $10.533,50$ & $<0,001$ & 100,00 \\
\multirow{2}{*}{ Estado geral de saúde } & Sim & 235 & 162,82 & & & 84,00 \\
\multirow{2}{*}{ Capacidade funcional } & Não & 116 & 216,53 & $8.928,00$ & $<0,001$ & 90,00 \\
& Sim & 235 & 155,99 & & & 75,00 \\
& Não & 116 & 202,76 & $10.526,00$ & $<0,001$ & 80,00 \\
& Sim & 235 & 162,79 & & & 76,00 \\
& Não & 116 & 202,67 & $10.536,50$ & 0,001 & 88,50 \\
& Sim & 235 & 162,84 & & & 77,00 \\
& Não & 116 & 187,99 & $12.239,00$ & 0,085 & 100,00 \\
& Sim & 235 & 170,08 & & & 100,00 \\
\hline
\end{tabular}

Teste U de Mann-Whitney

expuseram a agrotóxicos apresentaram piores escores totais de qualidade de vida.

A análise do estado geral de saúde (EGS) entre os indivíduos que usavam e não usavam agrotóxicos, sinalizou diferença estatisticamente significativa nessa relação $(\mathrm{U}=10536,50 ; p=0,001)$, ou seja, indivíduos que não usavam agrotóxicos apresentaram escores maiores $(X=88,50)$ e tendiam a ter com maior frequência um EGS melhor quando comparados aos que usavam agrotóxicos cujos escores eram menores $(X=77)$ (Tabela 5). Estes dados podem estar relacionados com os achados de Silva et al. ${ }^{26}$, que afirmam que os agricultores, em geral, desenvolvem as atividades de preparo e aplicação dos agrotóxicos em um cenário de misturas destes produtos, esforço físico e temperaturas elevadas. Neste aspecto, os resultados apresentados nos escores do item limitação por aspectos físicos $(\mathrm{U}=11183,50 ; p=0,003)$ identificaram que os usuários de agrotóxicos $(X=90)$ apresentaram mais limitações que os não usuários $(\mathrm{X}=100)$ como indicado na Tabela 5. Os escores relacionados à capacidade funcional não apresentaram significância $(p>0,05)$.

Em relação ao aspecto de Saúde Mental, os que não utilizavam agrotóxicos $(\mathrm{X}=80)$ revelaram tendência a apresentar com mais frequência um melhor rendimento neste aspecto quando comparados aos que utilizavam agrotóxicos ( $\mathrm{X}$ =76) $(\mathrm{U}=10526,00, \mathrm{p}<0,001)($ Tabela 5). Estes resultados corroboraram os achados de Zhang et al. ${ }^{29} \mathrm{em}$ trabalhadores chineses expostos a agrotóxicos com ideias suicidas, relacionando o uso destes ao aumento do risco na saúde mental, e a Tabela 5 também os revela sob o prisma da limitação por aspectos emocionais ( $U=10980,50, p$ $<0,001)$. Os dados encontrados guardaram relação com a presença de queixas de confusão mental, nervosismo, labilidade emocional, entre outras apresentadas em 52,48\% dos agricultores usuários de agrotóxicos em Atibaia (SP), descrito em levantamento de 101 prontuários médicos do Programa de Saúde da Família (PSF) de Rio $\mathrm{Abaixo}^{30}$. Desta maneira, os distúrbios de comportamento e os surtos psicóticos, quando medicados, podem estabelecer um nexo associativo de risco pela dupla intoxicação devido à interação de substâncias tóxicas diversas, agrotóxicos e medicamentos ${ }^{21}$.

Os achados apresentados revelam que os agravos à saúde da população de trabalhadores rurais do Povoado Colônia Treze expostos a agrotóxicos representam um fator de risco para uma qualidade de vida satisfatória, além disso, as per- 
das auditivas estão diretamente relacionadas com a exposição a este agente agressor.

\section{Conclusão}

Este estudo observou que os agricultores do Povoado Colônia Treze, usuários de agrotóxicos, apresentaram piores escores de qualidade de vida quando comparados com aqueles que não os utilizaram. Outro aspecto relevante foi que o uso de agrotóxico e sua classe toxicológica interferiram de maneira impactante no grau de perda auditiva apresentada nos mesmos.

Este estudo revelou que houve associação dos efeitos dos agrotóxicos sobre a saúde auditiva e a qualidade de vida perante este grupo populacional, estes resultados poderão subsidiar as políticas públicas com relação aos riscos à saúde e ao ambiente.

\section{Colaboradores}

TRR Sena trabalhou na participação da pesquisa de campo e na redação do artigo, na pesquisa de campo e interpretação dos dados, na discussão e redação final do artigo, na revisão crítica do artigo e na metodologia; MM Vargas trabalhou na orientação e supervisão da pesquisa de campo; e CCC Oliveira trabalhou na concepção e delineamento, análise e interpretação dos dados e na aprovação da versão a ser publicada. 


\section{Referências}

1. Miller GT. Ciência ambiental. 11 $1^{\text {a }}$ Edição. São Paulo: Thomson Learning; 2007.

2. Brasil. Ministério da Saúde (MS). Plano Integrado de Vigilância em Saúde de Populações Expostas a Agrotóxicos. Brasília: Editora do Ministério da Saúde; 2009.

3. Moreira JC, Jacob SC, Peres F, Lima JS, Meyer A, Oliveira-Silva JJ, Sarcinelli PN, Batista DF, Egler M, Faria MVC, Araújo AJ, Kubota AH, Soares MO, Alves SR, Moura CM, Curi R. Avaliação integrada do impacto do uso de agrotóxicos sobre a saúde humana em uma comunidade agrícola de Nova Friburgo, RJ. Cien Saude Colet 2002; 7(2):299-311

4. Figueiredo GM. Efeitos na saúde de trabalhadores expostos a longo prazo a agrotóxicos atendidos no ambulatório de toxicologia do Hospital das Clínicas da UNICAMP nos anos de 2006 e 2007 [dissertação]. Campinas (SP): Unicamp; 2009.

5. Siqueira LS, Kruse MHL. Agrotóxicos e saúde humana: contribuição dos profissionais do campo da saúde. Rev Esc Enferm USP 2008; 42(3):584-590.

6. Brasil. Ministério da Saúde (MS). Protocolo de Atencão à Saúde dos Trabalhadores Expostos a Agrotóxicos. Brasília: Editora do Ministério da Saúde; 2006.

7. Manjabosco CW, Morata TC, Marques JM. Perfil audiométrico de trabalhadores agrícolas. Arq Int Otorrinolaringol 2004; 8(4):285-295.

8. Soares WL, Porto AO. Estimating the social cost of pesticide use: An assessment from acute poisoning in Brazil. Ecol Econ 2009; (68):2721-2728.

9. World Health Organization (WHO). Public Health Impact of Pesticides Used in Agriculture. Geneva: WHO; 1990.

10. Bielefeld EC, Hu BH, Harris C, Henderson D. Damage and threshold shift resulting from cochlear exposure to Paraquat-generated superoxide. $J$ Occup Med Toxicol 2009; 207(1-2):35-42.

11. Hoshino ACH, Pacheco-Ferreira H, Taguchi CK, Tomita S, Miranda MF. Ototoxicity study in workers exposed to organophosphate. Braz J Otorhinolaryngol 2008; 74(6):912-918.

12. Bernardi APA. Exposição ocupacional a ruído e solventes e alterações auditivas periféricas e centrais [tese]. São Paulo (SP): Universidade de São Paulo; 2007.

13. Morata TC, Little B. Suggested guidelines for studying the combined effect of occupational exposure to noise and chemicals on hearing. Noise Health 2002; 4(14):73-87.

14. Instituto Brasileiro de Geografia e Estatística (IBGE). Censo agropecuário. Rio de Janeiro: IBGE; 2006.

15. Silva MS, Antoniolli AR, Batista JS, Mota CN. Plantas medicinais utilizadas nos distúrbios do trato gastrointestinal no Povoado Colônia Treze, Lagarto-SE. Acta Bot Bras 2006; 20(4):815-829.

16. Santos CM, Antônio EMM. I Caderno de pesquisa histórica. Lagarto: FJAV; 2008.

17. Teixeira CF, Augusto LGS, Morata TC. Saúde auditiva de trabalhadores expostos a ruído e inseticidas. Rev Saude Publica 2003; 37(4):417-423.
18. Instituto Brasileiro de Geografia e Estatística (IBGE). Relação de Localidades por Município: Atualização da base cartográfica. Rio de Janeiro: IBGE; 2007.

19. Merluzzi F, Cornacchia L, Parigi G, Terrana T. Metodologia di esecuzione del controllo dell'udito dei lavoratori esposti a rumore. Nuovo Arch Ital Otol 1979; 7:695-714.

20. Ciconelli RM, Ferraz MB, Santos W, Meinão I, Quaresma MR. Tradução para a língua portuguesa e validação do questionário genérico de avaliação de qualidade de vida SF-36 (Brasil SF-36). Rev Bras Reumatol 1999; 39(3):143-150.

21. Levigard YE, Rozemberg B. A interpretação dos profissionais de saúde acerca das queixas de "nervos” no meio rural: uma aproximação ao problema das intoxicações por agrotóxicos. Cad Saude Publ 2004; 20(6):1515-1524

22. Instituto de Pesquisa Econômica Aplicada (IPEA). Pesquisa Nacional por Amostra de Domicílios (PNAD) de 2008. Brasil: IPEA; 2008.

23. Sergipe. Secretaria de Estado de Agricultura e Desenvolvimento Agrário, Programas e Projetos (SEADAPP). Agricultura: realidade e perspectivas. Aracaju: SEADAPP; 2009.

24. Bittar RSM, Bottino MA, Zerati FE, Moraes CLO, Cunha AU, Bento RF. Prevalência de alterações metabólicas em pacientes portadores de queixas vestibulares. Rev Bras Otorrinolaringol 2003; 69(1):64-68.

25. Damalas CA, Eleftherohorinos IG. Pesticides exposure safety issues, and risk assessment indicators. Int J Environ Res Public Health 2011; 8(5):1402-1419.

26. Silva JM, Novato-Silva E, Faria HP, Pinheiro TMM. Agrotóxico e trabalho: uma combinação perigosa para a saúde do trabalhador rural. Cien Saude Colet 2005; 10(4):891-903.

27. Soares W, Almeida RMVR, Moro S. Trabalho rural e fatores de risco associados ao regime de uso de agrotóxico em Minas Gerais., Brasil. Cad Saude Publ 2003; 19(4):1117-1127.

28. Schlindwein VLDC. Dor e sofrimento oculto: a desproteção social dos trabalhadores do fumo. Barbaroi 2010; (32):82-97.

29. Zhang J, Stewart R, Philips M, Shi Q, Prince M. Pesticide exposure and suicidal ideation in rural communities in Zhejiang province, China. Bull World Health Organ 2009; 87(10):745-753.

30. Lima PJP. Possíveis doenças físicas e mentais relacionadas ao manuseio de agrotóxicos em atividades rurais nas região de Atibaia, SP/ Brasil [dissertação]. São Paulo: Universidade de São Paulo; 2008.

Artigo apresentado em 28/11/2011

Aprovado em 20/02/2012

Versão final apresentada em 04/03/2012 
